\title{
WILEY-VCH
}

DOI: $10.1002 /(($ please add manuscript number $))$

Article type: Full Paper

\section{A full vacuum approach for the fabrication of hybrid white light emitting thin films and wide-range in situ tunable luminescent microcavities}

Youssef Oulad-Zian, Juan Ramon Sanchez-Valencia,* Manuel Oliva, Julian Parra-Barranco, Maria Alcaire, Francisco J. Aparicio, Ana Mora-Boza, Juan Pedro Espinos, Francisco

Yubero, Agustin R. Gonzalez-Elipe, Angel Barranco and Ana Borras*

Nanotechnology on Surfaces Lab. Materials Science Institute of Seville (ICMS, CSIC-US), Avd. Americo Vespucio 49, Seville, 41092, Spain.

E-mail: jrsanchez@icmse.csic.es; anaisabel.borras@icmse.csic.es;

Keywords: GLAD, Rubrene, photonic crystals, Bragg microcavities, white light emission

\section{ABSTRACT}

In this article we show the fabrication by a dry approach at mild temperature $\left(<150^{\circ} \mathrm{C}\right)$ of a photoluminescence white light emitting hybrid layer. The white light emitter is obtained by evaporation of two photoluminescent small molecules, a blue (1,3,5-triphenyl-2-pyrazoline TPP) and an orange (rubrene) dye within the porous of $\mathrm{SiO}_{2}$ host film fabricated by glancing angle deposition (GLAD). Fluorescencence (Föster) resonant energy transfer between the two organic dyes allows the emission of the combined system upon excitation of the TPP molecule at wavelength of $365 \mathrm{~nm}$. The distribution of the organic molecule within the host layer is analysed as a function of the substrate temperature and vacuum conditions and the required conditions for the white emission determined by finely controlling the TPP:Rubrene ratio. The full vacuum processing of the hybrid layers provides a straightforward route for the incorporation of the white light emitters as optical defect within one-dimensional Bragg microcavities. As consequence, directional emission of the system is achieved which allows the development of wide-range in situ tunable photoluminescent devices. 


\section{WILEY-VCH}

Organic nanomaterials have been widely used for the fabrication of photonic devices such as photovoltaic cells, [1] sensors[2] and light emitters[3] taking advantage of the large variety of photonic-active molecules, the control on their functionalities through molecular design and the potential for low cost synthesis and manufacturing. Concretely, the incorporation of organic light-emitting materials as active components in electroluminescence devices has demonstrated a great perspective for the fabrication of organic light emitting diodes (OLED) for light weight, low cost and low power consumption colour displays.[1,3] Specifically, organic white- and whitish-light emitters are one of the most critical components in fullcolour or backlight source portable displays.[1-3] White-light emitters are commonly fabricated by combining different emitters (red/blue/green or blue/orange) covering the visible range from 400 to $700 \mathrm{~nm}$ and numerous works have reported about electroluminescence devices based on multilayer stacked structures of blue/green and redish/orange emitters.[3-10] Thus, the orange dye dopant rubrene has been one of the most widely used molecules for the development hybrid-white organic light emitting diodes (HWOLEDs).[11] Altogether to the development of organic and hybrid electroluminescent white emitters, their photoluminescent counterpart has also undergone interesting advances in terms of enhancement in quantum yield and control on the colour emission.[12-17] On the other hand, it has recently been proposed the incorporation of electroluminescent organic light emitters within an optical vertical microcavity as a feasible route for the improvement in colour quality.[18] Desirable outputs from the coupling of the light emission with the cavity mode are the narrowing of the emission band and improvement of the efficiency, leading to purer colour and enhanced-efficiency organic light emitters. The integration of luminescent media within vertical microcavities as well allows to finely adjust the emission output as a function of the different parameters controlling the characteristics of the photonic structure (cavity thickness, refractive index contrast and periodicity).[18,19] Furthermore, these architectures are the base for the development of in situ tuneable lighting systems. In some 


\section{WILEY-VCH}

complex designs, the colour tuneability is achieved by electro-mechanical actuation on the microcavity thickness,[20] meanwhile other approaches take advantage of the angular dependence of the optical properties of the microcavity.[21] The latter represents a simple way to tune the output wavelength as a function of the device angle.[22] However in all these systems (ex situ adjusted and in situ tuneable luminescent vertical microcavities) the operation range is limited to the spectral region where the active medium emits. All these antecedents have motivated the development of electroluminescent white emitting materials for the implementation in optical microcavities. However, the application of this approach to photoluminescent emitters is almost unexplored and even rarer in the case of white emitters. The main reason is likely due to the difficult realization of an optical microcavity able to locate the emitters (without distorting the cavity) and with high transparency to allow the excitation of the organic dyes. Within this context, we present herein the development of a new family of hybrid white light emitters prepared by a full vacuum deposition approach. The white emission is obtained by incorporation of two photoluminescent small molecules, a blue (1,3,5-triphenyl-2-pyrazoline TPP) and an orange (rubrene) emitter (see chemical structure in Figure 1 a) by physical vapour deposition (PVD) using as host a $\mathrm{SiO}_{2}$ thin film fabricated by glancing angle vacuum deposition (GLAD). This type of films offer unique host possibilities for the incorporation of active molecules, metal nanoparticles, or quantum dots for the development of photonic gas sensors, information encoding or biodetectors.[23-41] In most of these applications the incorporation of the photonic or functional active component(s) requires the intervention of wet chemical processes.[23-31,34,42-45] In contrast, the present work aims primarily at developing a "dry" evaporation-based procedure for the incorporation of several organic dye molecules into the pores of inorganic host thin films. The success of the approach has been demonstrated by sequentially incorporating TPP and Rubrene (Rub) evaporated molecules in the interior of a $\mathrm{SiO}_{2}$ porous thin film, which, in the form of a transparent layer, provides robustness, high optical quality and optimal processing capability. 


\section{WILEY-VCH}

From now on we will refer such hybrid films as TPP:Rub@ $\mathrm{SiO}_{2}$. In a second term, physical vapour deposition has been used to integrate the hybrid layers as an optical defect within a 1D photonic Bragg microcavity structure formed by the stacking of inorganic layers of different refraction indices.[18,46,47] This hybrid system behaves as an angular dependent light source where tuneable wavelength fluorescence is emitted within a very narrow angular window.

The "host" material in the hybrid layers consists on tilted nanocolumnar $\mathrm{SiO}_{2}$ thin films characterized by the porous nanostructure depicted in Figure 1 b) (see also Supporting Information Section S1 and Figure S1) and a high transparency as shown in Figure $1 \mathrm{~d}$ ) (black curve). A key feature of the process is the homogeneous incorporation of dispersed molecules within the host films, avoiding a crystal aggregation that might hamper the high fluorescence and intermolecular energy transfer phenomena required to induce white light emission. This can be proved by the scanning electron microscope observation of the films before (Figure 1b) and after evaporation (Figure 1c) and the UV-vis transmission (Figure 1d) and luminescent (Figure 1e) spectra of hybrid thin films incorporating separately TPP and rubrene molecules. The optical behaviour of these films is characterized by the typical absorption (Figure $1 \mathrm{~d}$ ) bands of dispersed TPP (blue curve) and rubrene (orange) molecules at ca. 360 and $500 \mathrm{~nm}$ respectively,[48] and their luminescence (solid line) and excitation (dashed) spectra reported in Figure 1e). Overlapping of the emission bands of TPP with the excitation bands of rubrene (shaded in grey in the Figure 1e) suggests the possibility to induce a Fluorescent (Förster) Resonant Energy Transfer (FRET).[49] Hence, we proceeded to the simultaneous incorporation of the two dye molecules by firstly evaporating rubrene at $150^{\circ} \mathrm{C}$ and then TPP at $60^{\circ} \mathrm{C}$. 
a)
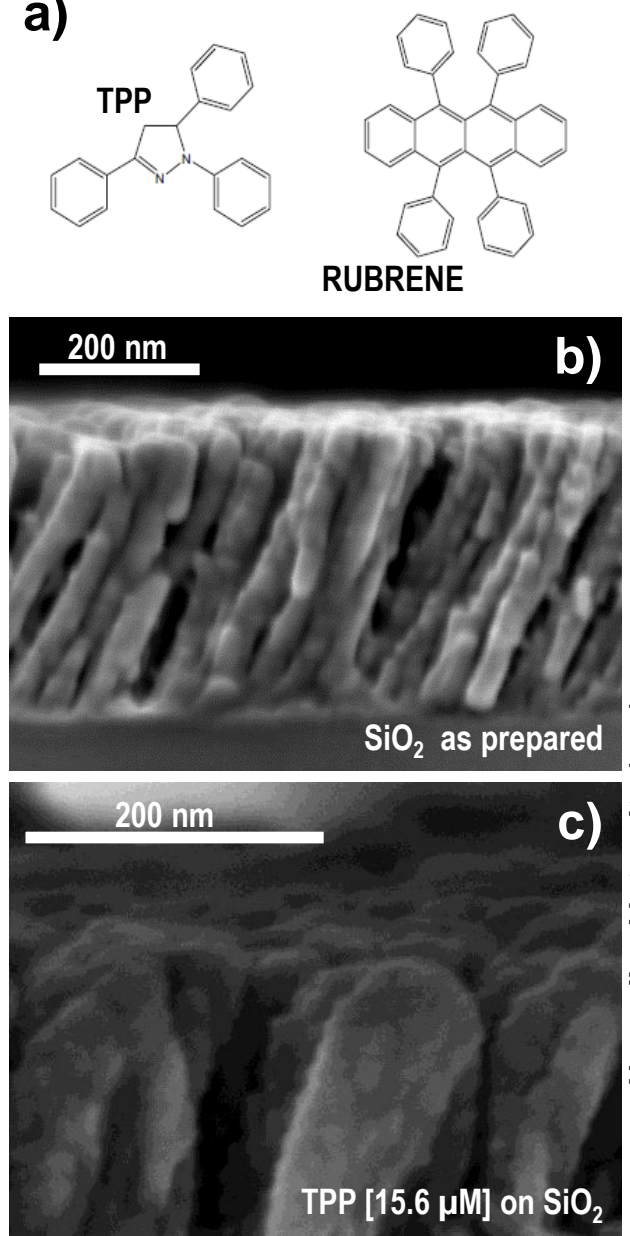

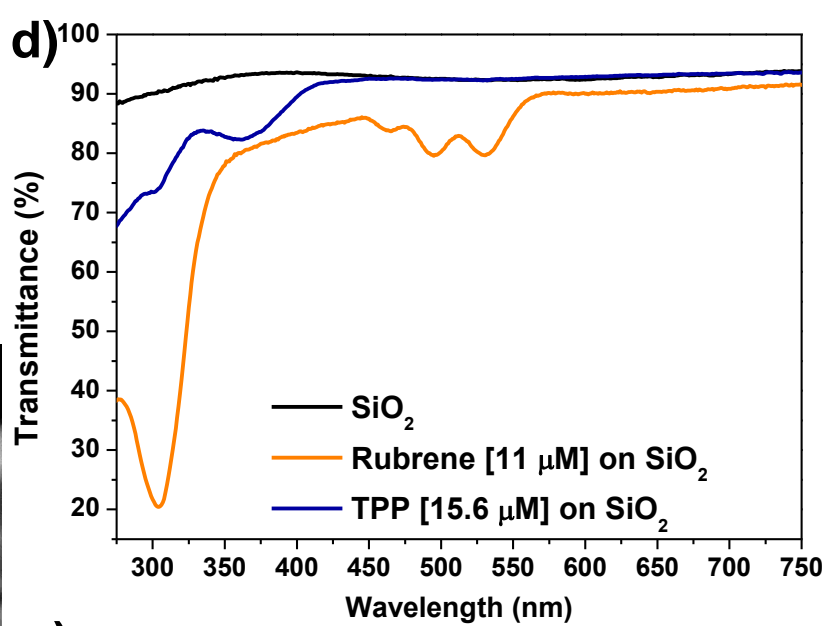

e)

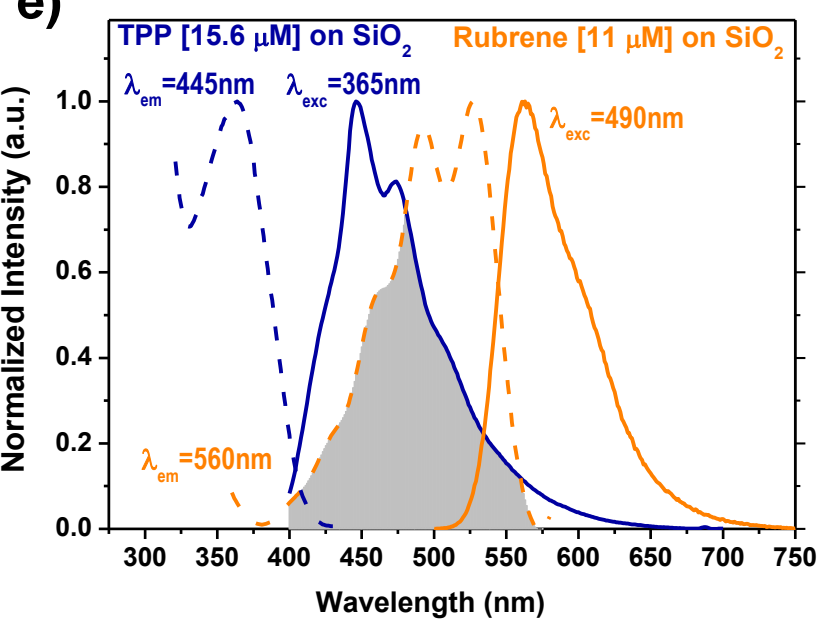

Figure 1. Microstructure and optical behaviour of the hybrid Rubrene@ $\mathrm{SiO}_{2}$ and $\mathrm{TPP} @ \mathrm{SiO}_{2}$ thin films. a) Chemical structure of the organic molecules; b) Characteristic cross section SEM micrograph of the $\mathrm{SiO}_{2}$ GLAD thin film used as hosts. c) A high magnification cross section view of the of the hybrid TPP@ $\mathrm{SiO}_{2}$ film showing the homogeneous incorporation of the dye without formation of organic crystals on the $\mathrm{SiO}_{2}$ surface. d) Transmission spectra of single molecule hybrid films as labelled, the black line corresponds to the bare $\mathrm{SiO}_{2}$ film used as host. e) Fluorescence emission (solid line) and excitation (dashed line) of the Rubrene@ $\mathrm{SiO}_{2}$ and $\mathrm{TPP} @ \mathrm{SiO}_{2}$ thin films as indicated. The equivalent concentration (see Experimental Section and Table S1) of organic molecules deposited in c) and d) were 15.6 and $11 \mu$ mols for TPP and Rubrene, respectively. 
Figure 2 a) shows the fluorescence emission spectra of a series of hybrid thin films with different combinations of TPP:Rubrene embedded in porous $\mathrm{SiO}_{2}$ films using excitation wavelengths at $350 \mathrm{~nm}$ for TPP and TPP:Rubrene and $490 \mathrm{~nm}$ for the pure rubrene systems, respectively (see Table S1 for the equivalent concentration and percentage of rubrene in each case). Despite the small amount of rubrene in the samples, this series of spectra (hereafter samples will be referred by the percentage of rubrene) are characterized by a very intense rubrene emission when illuminating through the excitation band of TPP. The emission intensity progressively increases with the amount of incorporated rubrene and is hardly detected when no TPP molecules are present in the films. The evolution of the emission intensities as a function of the relative amount of the two molecules (Figure 2 a) proves that a robust and non-dispersive white light emitter thin film can be prepared by properly controlling the relative intensities of the TPP and rubrene emission bands. Besides, this graph also shows the development of the of the rubrene emission band when TPP molecules are present in the nanocolumnar films, particularly when comparing the strong rubrene emission of sample $4.5 \%$ with the weak fluorescence signal of sample $100 \%$ Rub. Provided that both samples present the same amount of rubrene, this comparison clearly indicates that the rubrene emission is much more efficiently excited through the TPP band. Hence, this phenomenon must be accounted for by a non-radiative intermolecular fluorescence resonant energy transfer (IFRET) process between TPP/Rubrene pairs.

The colour coordinates $(\mathrm{x}, \mathrm{y})$ extracted from the fluorescence spectra of these TPP:Rubrene@ $\mathrm{SiO}_{2}$ hybrid films depict a straight line between the colours blue (pure TPP, 0\% Rub) and yellow (pure rubrene, 100\% Rub) (see Figure 2b). In this line profile, white light emission was found for $2.7 \%$ of rubrene (Figure $2 \mathrm{c}-\mathrm{d}$ ). The films with this particular rubrene concentration depict the absorbance (dashed grey line in c), excitation (c) and emission (d) spectra reported in Figure 2. Thus, the photograph in (e) demonstrates that these films, otherwise transparent and practically non-coloured, behave as white emitters when excited 


\section{WILEY-VCH}

with UV light (see also Figure 2 b, 2.7\% Rub). Meanwhile, films with 2.2/4.5\% of rubrene yield bright blue/orange colours (c.f. photographs in Figure 2 e) under illumination with $\lambda=$ $365 \mathrm{~nm}$ (see also the coordinate colours in Figure 2b). In this film white light emission results from the similar intensities of the TPP and Rubrene fluorescence bands at, respectively, 450 and $550 \mathrm{~nm}$ (Figure $2 \mathrm{~d}$ ) upon excitation with $\lambda_{\mathrm{exc}}=365 \mathrm{~nm}$, i.e. the excitation wavelength of TPP (red line in Figure $2 \mathrm{c}$ ). For the same sample, excitation at the wavelength corresponding to rubrene $\left(\lambda_{\mathrm{exc}}=490 \mathrm{~nm}\right)$ yields a weaker fluorescence emission centred at $550 \mathrm{~nm}$ typical of the orange dye.

The absorbance spectrum of the white light emitter is dominated by a band at around $370 \mathrm{~nm}$ due to TPP and an almost negligible contribution at around $500 \mathrm{~nm}$ due to Rubrene. By contrast, in the excitation spectrum recorded by measuring the fluorescence emission at 560 $\mathrm{nm}$ (black line in Figure 2c) the excitation bands associated to both dyers are clearly distinguished. Moreover, the similar shape and position of the rubrene excitation band in the white emitter and the absorption band recorded for a Rubrene @ $\mathrm{SiO}_{2}$ reference sample (inset of Figure 2c) orange line) supports that the rubrene and TPP molecules are homogeneously dispersed within the matrix of the white emitter sample[49] (additional considerations about the homogeneous distribution of the molecules within the GLAD sample are presented in the Supporting Information S2 and Figure S2) and in good agreement with the literature $[48,50,51]$ that the white emission phenomenon must be accounted for by a nonradiative intermolecular fluorescence resonant energy transfer (IFRET) process between TPP/Rubrene pairs. A further evidence is supplied by the similar TPP excitation bands in Figure 2c) recorded by monitoring the fluorescence emissions at 560 and $450 \mathrm{~nm}$. 


\section{WILEY-VCH}

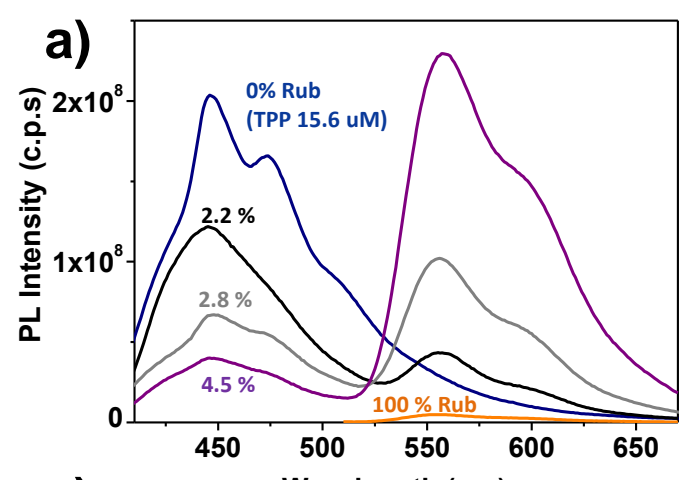

b)
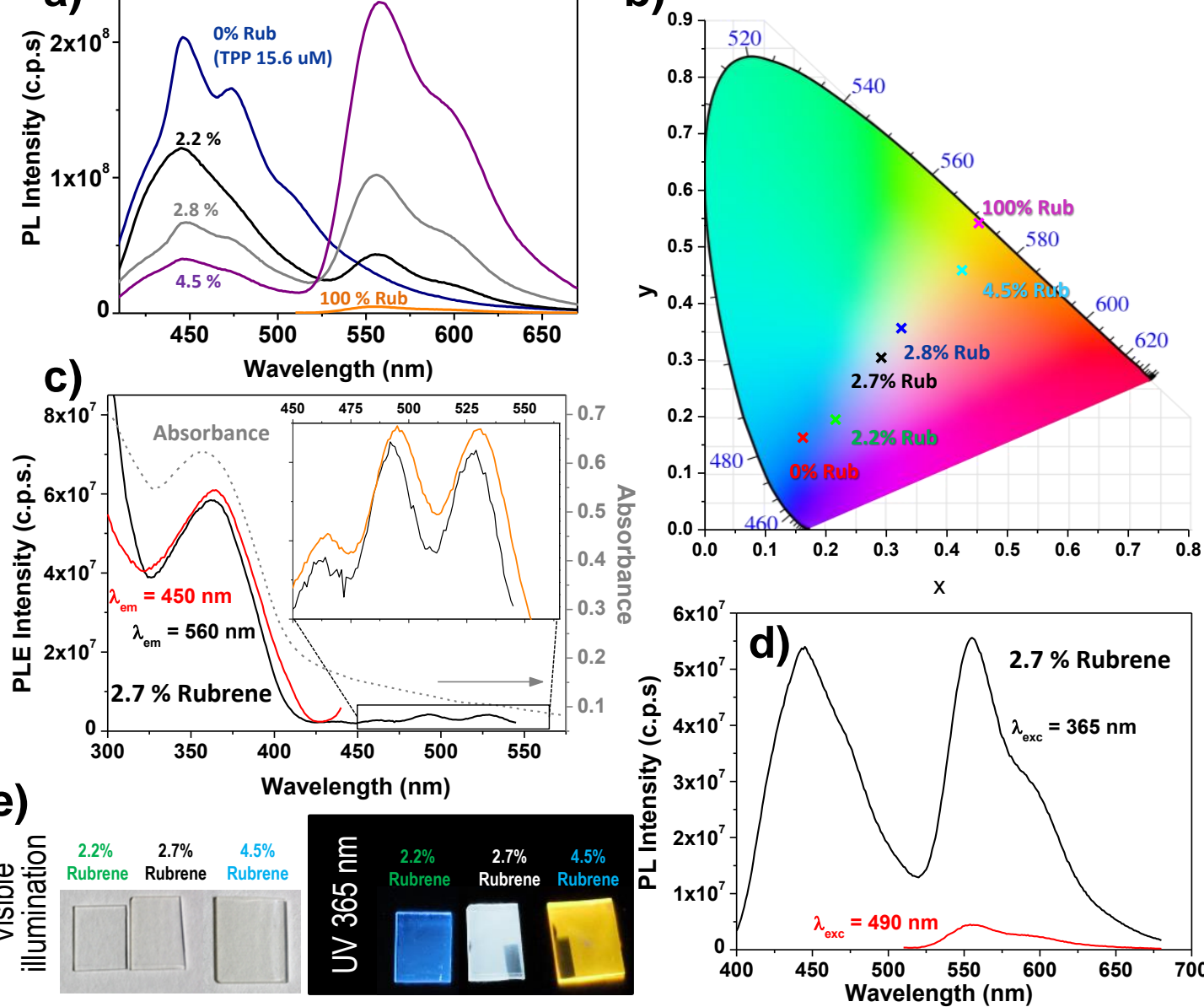

Figure 2. Optical behaviour of white light emitter and related hybrid thin films. a)

Fluorescence emission spectra recorded for thin films with variable TPP:Rubrene ratios (expressed as percentage of Rubrene) incorporated into the $\mathrm{SiO}_{2}$ GLAD thin films-as labelled. Excitation wavelengths selected at $365 \mathrm{~nm}$ for the TPP and TPP:Rubrene samples and $490 \mathrm{~nm}$ for the Rubrene. b) Coordinate colours $\mathrm{x}, \mathrm{y}$ corresponding to different hybrid samples extracted from the fluorescent emission shown in a) and d). c-d) Absorbance (c, dashed grey line), excitation (c) and emission spectra (d) of the thin film with $2.7 \%$ Rubrene behaving as white emitter. Inset in c) shows a comparison of the excitation fluorescent profile (monitoring the emission at $560 \mathrm{~nm}$ ) of the sample with $2.7 \%$ Rubrene with the absorption bands of a 100 $\%$ Rubrene film. e) Photographs of the representative samples excited with visible (left) and UV light (right). 


\section{WILEY-VCH}

In this work we aim to demonstrate that the high level of processing of the hybrid TPP:Rubrene@ $\mathrm{SiO}_{2}$ and straightforward compatibility of the procedure with well-established vacuum methodologies for the deposition of optical thin films allow a "dry" approach to the fabrication of angular and wavelength tuneable emission devices. Thus, we have incorporated the TPP:Rubrene@ $\mathrm{SiO}_{2}$ thin film as an optical defect within a Bragg microcavity (MC) structure (Figure 3 a-b). The hybrid film is deposited in between of two one dimensional photonic crystals (1DPC) formed by a multilayer periodic structure of compact $\mathrm{TiO}_{2}$ and $\mathrm{SiO}_{2}$. Considering the wide emission range of the developed material, the Bragg microcavity was designed ad hoc to exhibit an optical band gap extended along almost all the whole visible region (450-650 nm) with two optical windows in the fluorescence region of the TPP/Rubrene system and transparent at the illumination wavelength of TPP (around $365 \mathrm{~nm}$ ). It is worth stressing that the realization of the coupling of the transmission of the MC with the organic emission is a two-fold remarkable achievement. Firstly, because the controlled vacuum process permits the incorporation of the organic dyes only in the porous layer acting as defect without infiltration of the 1DPC bottom-mirror and the vacuum and low temperature deposition of the top-1DPC does not alter the properties of the TPP:Rubrene@ $\mathrm{SiO}_{2}$ defect. Secondly, the excitation of the photoluminescent system is possible by illumination at $365 \mathrm{~nm}$ of the TPP through the $\mathrm{SiO}_{2} / \mathrm{TiO}_{2}$ multilayers due to the designed transparent window within this region (IW in Figure $3 \mathrm{c}$-d). The photonic structure consisted of 14 alternant compact layers of $\mathrm{TiO}_{2}(\approx 80 \mathrm{~nm}, \mathrm{n}=2.1) / \mathrm{SiO}_{2}(\approx 70 \mathrm{~nm}, \mathrm{n}=1.4)$ and a central TPP:Rubrene $@ \mathrm{SiO}_{2}$ layer of $420 \mathrm{~nm}$ (see Figure $3 \mathrm{a}-\mathrm{b}$ and Figure S3 for the simulation of the optical transmittance of the MC). Figure $3 \mathrm{c}$ ) demonstrates the effective coupling of the $\mathrm{MC}$ with the fluorescence emission for two different hybrid system, a white light (2.7\% of Rubrene, blue line) and a yellowish (2.8\% of Rubrene, orange line) TPP:Rubrene@ $\mathrm{SiO}_{2}$ emitters. This $\mathrm{MC}$ presents two resonant peaks at 480 (TW1) and $600 \mathrm{~nm}$ (TW2) located within the luminescence bands of TPP and Rubrene. The transmission of the MC (i.e., through the illumination window, IW) 


\section{WILEY-VCH}

at $365 \mathrm{~nm}$ corresponding to the TPP excitation is about the $45 \%$. The illumination of the MCs at $365 \mathrm{~nm}$ results in the normalized luminescence spectra of the Figure $3 \mathrm{~d}$ ) recorded at $0^{\circ}$ (see schematic on the geometry as inset in panel f) and Figure S4 showing the angular dependency of the MC optical transmittance). The common effect in both emitters is the narrowing of the emission bands and the shift of the emission maxima towards the centre of the transmission windows. In addition to the peaks related to TW1 and TW2 it appears a broader and smaller band at approximately $440 \mathrm{~nm}$ that might be linked to slight transmission of the MC at this wavelength (the transmission at $440 \mathrm{~nm}$ is around 40\%). By selecting the peak at $580 \mathrm{~nm}$ for the $2.8 \%$ Rubrene, the width of the emission is reduced to a third part, from FWHM $=57 \mathrm{~nm}$ in the hybrid layer to $20 \mathrm{~nm}$ within the microcavity. In the case of the white emitter, the width of the same peak goes from $60 \mathrm{~nm}$ to $15 \mathrm{~nm}$ once implemented in the MC. Thus, following the conclusions in reference 18 the integration of the hybrid films into the Bragg microcavity improves the quality of the colour in terms of purity or saturation. Nevertheless, the most interesting results develop about the angular behaviour of the luminescent MC. On the one hand, Figure S5 shows the luminescence emission of the $2.8 \%$ Rubrene MC for two different orientations respect to the excitation (see also schematic in Figure $3 \mathrm{f}$ ). The spectrum acquired in normal configuration (at " 0 " " in the scheme) depicts the shape already mentioned in Figure $3 \mathrm{~d})$. Interestingly, when the orientation of the MC is tuned to " $60^{\circ}$ " the emission vanishes even though the transmittance of the MC at this latter angle yet corresponds to the $45 \%$ (Figure S5). This result indicates the coupling between the TPP:Rubrene@ $\mathrm{SiO}_{2}$ with the Bragg microcavity provides a directional luminescence. In general, fluorescence emission is non-directional and sophisticated procedures are applied when directionality is a key issue.[50-52] Directional emission is extremely useful since it permits to collect all the fluorescence light at a certain direction (otherwise isotropically distributed) improving the fluorescent collection efficiency for applications in biosensors or single-molecule detection.[53,54] In addition, the angular control in the emission can be determinant for the 


\section{WILEY-VCH}

development of colour tuneable lighting systems.[21] Fluorescence angular control has been recently achieved by coupling the spontaneous emission of fluorescent molecules to optical antennas[54] and by embedding the fluorescence material within optical fibers.[55] Incorporation of fluorescent materials within photonic structures represents a more reliable way to control both the spectral and spatial characteristics of the emission output.[21,52,56]

On the other hand, if the combination of dyes embedded in the defect corresponds to the white emitters films the angular dependence of the luminescent $\mathrm{MC}$ becomes more complex. Figure 3 e) presents the experimental (bottom) and simulated (top) photoluminescence spectra as a function of the collection angle of the $2.7 \%$ Rubrene MC. The simulations have been obtained through convolution of the MC transmittance as a function of the collection angle (Figure S3) and the luminescence emission shown in Figure 2 d). Thus, when the device is excited at $\lambda_{\text {exc }}=365 \mathrm{~nm}$, the fluorescence emission depicts a strong angular dependency. At $0^{\circ}$, the white emission of this angular dependent emitter was slightly shifted to the yellow (see colour coordinates in Figure 3f) due to the blocking of blue emission by the photonic crystal. Interestingly, taking advantage of the wide range emission of the $2.7 \%$ rubrene layer, it is possible to finely tune the emitted colour of the MC by a simple adjustment of the MC angle. Hence, for a given composition of the hybrid layer, the emission colour can be selected in situ by rotation of the MC respect to the detector. As far as we know, these results show for the first time a full vacuum approach to the implementation of a hybrid white light emitter fluorescent layer into an optical microcavity as well as demonstrate the suitable modulation of the fluorescence signal according to the optical properties of the photonic structure. 
WILEY-VCH
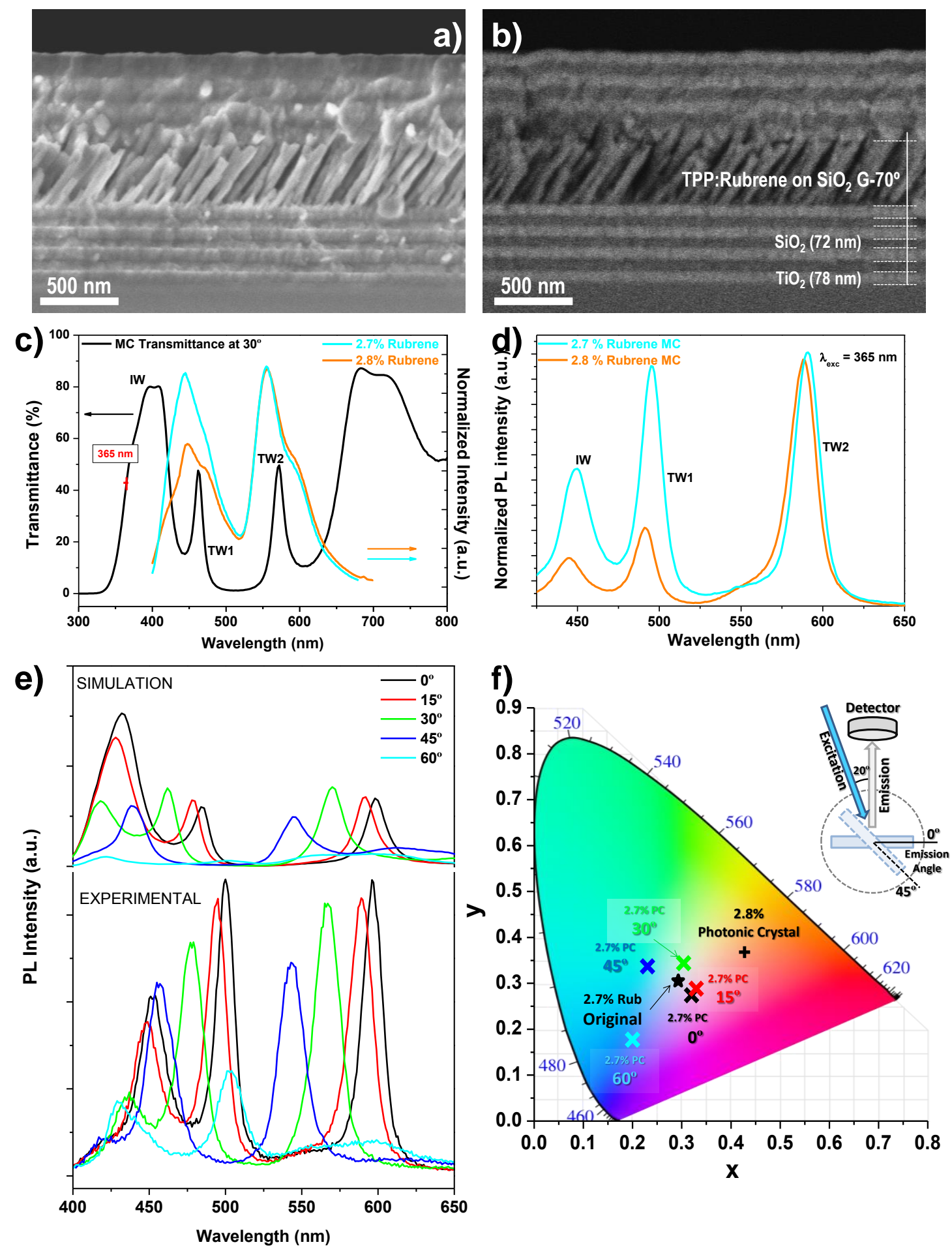

Figure 3. Incorporation of hybrid white emitter thin films into a Bragg microcavity structure.

Cross section SEM micrographs of the devices under secondary (a) and backscattered (b) electrons. In this case the stack layers system corresponds to $78 \mathrm{~nm}-\mathrm{TiO}_{2}($ refractive index $\mathrm{n}(550 \mathrm{~nm})=2.1)$ and $72 \mathrm{~nm}-\mathrm{SiO}_{2}(\mathrm{n}(550 \mathrm{~nm})=1.4)$ and the $2.7 \%$ Rubrene hybrid film incorporated into $\mathrm{SiO}_{2} \mathrm{GLAD}-7^{\circ}$. Deposition of the top stack layers was carried out by 


\section{WILEY-VCH}

electron beam evaporation at normal incidence using a cooling system in order to avoid sublimation of TPP during the process. c) Normalized luminescence spectra for the $2.7 \%$ and $2.8 \%$ Rubrene @ $\mathrm{SiO}_{2}-70^{\circ}$ overlapping to the UV-Vis transmission of the Bragg microcavity incorporating the defect. e) Simulated (top) and experimental (bottom) emission spectra recorded at normal and off-normal geometries for the $2.7 \%$ Rubrene@ $\mathrm{SiO} 2-70^{\circ} \mathrm{Bragg}$ microcavity structure. Simulated spectra are corrected by the UV transmission value at 365 nm.

In this work we have developed an full vacuum methodology for the fabrication of fluorescent emitter hybrid thin films and photonic structures which, due to their outstanding optical characteristics and the absence of any light dispersing behaviour, can be directly utilized for specific applications where the control over the colour and angular dependence of the emitted light are key features. Besides the robustness of the processing technique and final devices obtained, the method provides the possibility of incorporating in a tailored way two different dye molecules and to control the Fluorescent Resonant Energy Transfer excitation process operating between them to achieve fluorescent white light emitters. The incorporation of these fluorescent thin film layers into a Bragg microcavity photonic structure has enabled the fabrication of directional luminescent emitters and microcavities with wide-range in situ colour-tuning capabilities. Among the advantages of the developed methodology it is worthy to specifically address the following characteristics: i) all the procedures involved are founded on physical vapour deposition processes, i.e. solvent-less environmentally friendly protocols; ii) the solvent-less character of the methodology allows the infiltration of different molecules independently of their solubility. Such advantage is critical when comparing this methodology with other stablished wet-approaches. Also in this sense, our methodology permits to control the location of the infiltrated molecules within the mesoporous host layer which makes 


\section{WILEY-VCH}

possible its direct incorporation in photonic structures as the Bragg microcavity reported herein; iii) the method is general from the point of view of organic and inorganic counterparts in the hybrid system. In fact, conclusions in terms of substrate temperature requirements for the diffusion of the molecules, sequential sublimation approach for the combination of two or more different dyes into the same host layer and integration of the hybrid active layers into photonic devices are straightforwardly extensible to an ample variety of vacuum processable small molecules and different composition of the porous layer acting as host. This general character is also applicable to the host material. Thus, the solvent-less operation at low and mild temperatures makes the procedure fully compatible with the use of thermal/solvent sensible organic flexible substrates as polymers or cellulose.

\section{Experimental Section}

$\mathrm{SiO}_{2}$ GLAD thin films were prepared on quartz and silicon substrates by electron evaporation at zenithal angles, $\alpha$, of $70^{\circ}$ and $80^{\circ} .[23-31,33,34]$ In the standard conditions, a typical distance of $80 \mathrm{~cm}$ separates the evaporation crucible and the substrate. Hybrid thin films were obtained by evaporating TPP and Rubrene molecules in a Knudsen cell over the host thin films kept at $150{ }^{\circ} \mathrm{C}$ for Rubrene and $60^{\circ} \mathrm{C}$ for TPP. Argon at a pressure of $2 \times 10^{-2}$ mbar was dosed to the chamber during the organic molecules deposition. The amount of deposited material, indirectly controlled by means of a quartz crystal monitor placed close to the substrate, is referred in the Table S1 as nominal thickness. The amount of molecules was estimated by the evaluation of the absorption bands of the hybrid films (after a background subtraction) and its direct comparison with the absorption of the corresponding solutions (TPP and Rubrene in ethanol and acetone, respectively). In this way we have followed a nondestructive optical method for the quantitative assessment of organic species within the device. The Table S1 shows the equivalent concentration for each molecule, i.e. the 


\section{WILEY-VCH}

absorption that would have the corresponding solution measured on a $1 \mathrm{~cm}$-path optical quartz cuvette.

When two dye molecules were incorporated into the films, the process was done sequentially. These crucibles, placed at the same distance of $8 \mathrm{~cm}$ from the substrate, were laterally moved to proceed to the evaporation when they were at the same vertical position and distance from the substrate.

Bragg microcavities structures, made up from the stacking of $\mathrm{SiO}_{2}$ and $\mathrm{TiO}_{2}$ individual layers, were prepared in the same evaporation set up by placing the substrates facing (i.e. normal orientation) to the electron beam evaporator. An oxygen pressure of $5 \times 10^{-4}$ mbar was kept during the evaporation of the $\mathrm{TiO}_{2}$ to ensure obtaining transparent and fully oxidized stoichiometric layers.[33-35] The in-situ control of the thickness of the individual stacked layers of $\mathrm{SiO}_{2}$ and $\mathrm{TiO}_{2}$ was done by means of a quartz crystal monitor placed besides the substrates.

Cross section and top-view SEM images of the hybrid thin films and Bragg microcavity devices was taken in a Hitachi S4800 field emission microscope. UV-Vis analysis of the samples was done in a Cary 100 spectrometer from Varian. Fluorescence spectra were recorded in a Jobin Yvon Fluorolog-3 spectrofluorometer using the front face configuration and grids of 4 and $2 \mathrm{~nm}$ for the excitation and emission monochromators.

\section{Supporting Information}

Supporting Information is available from the Wiley Online Library or from the author.

\section{Acknowledgements}

We thank the Junta de Andalucia (TEP8067, FQM-6900, and P12-FQM-2265), the Spanish Ministry of Economy and Competitiveness (MAT2013-40852-R and MAT2013-42900-P) and Abengoa Research S. L. (Framework Project) for financial support. 


\section{WILEY-VCH}

Received: ((will be filled in by the editorial staff))

Revised: ((will be filled in by the editorial staff))

Published online: ((will be filled in by the editorial staff))

[1] Y. S. Zhao, C. Di, W. Yang, G. Yu, Y. Liu, J. Yao, Adv. Funct. Mater. 2006, 16, 1985.

[2] M. D. Shirsat, T. Sarkar, J. Kakoullis, N. V. Myung, B. Konnanath, A. Spanias, A.

Mulchandani, J. Phys. Chem. C 2012, 116, 3845.

[3] S. Reineke, F. Lindner, G. Schwartz, N. Seidler, K. Walzer, B. Lüssem, K. Leo, Nature 2009, 459, 234.

[4] F. Steuber, J. Staudigel, M. Stössel, J. Simmerer, A. Winnacker, H. Spreitzer, F. Weissörtel, J. Salbeck, Adv. Mater. 2000, 12, 130.

[5] J. Kido, M. Kimura, K. Nagai, Science 1995, 267, 1332.

[6] R. H. Jordan, A. Dodabalapur, M. Strukelj, T. M. Miller, Appl. Phys. Lett. 1996, 68, 1192.

[7] H.-P. Lin, F. Zhou, J. Li, X.-W. Zhang, D.-B. Yu, L. Zhang, X.-Y. Jiang, Z.-L. Zhang, J. Ind. Eng. Chem. 2011, 17, 675.

[8] Y. Sun, N. C. Giebink, H. Kanno, B. Ma, M. E. Thompson, S. R. Forrest, Nature 2006, 440, 908.

[9] J. Liu, L. Chen, S. Y. Shao, Z. Y. Xie, Y. X. Cheng, Y. H. Geng, L. X. Wang, X. B. Jing, F. S. Wang, Adv. Mater. 2007, 19, 4224.

[10] J. Kido, K. Hongawa, K. Okuyama, K. Nagai, Appl. Phys. Lett. 1994, 64, 815.

[11] C. H. Chuen, Y. T. Tao, Appl. Phys. Lett. 2002, 81, 4499.

[12] N.-C. Chen, C.-C. Liao, C.-C. Chen, W.-T. Fan, J.-H. Wu, J.-Y. Li, S.-P. Chen, B.-R. Huang, L.-L. Lee, Nanoscale Res. Lett. 2014, 9, 569.

[13] A. M.-C. Ng, A. B. Djurišić, K.-H. Tam, W.-M. Kwok, W.-K. Chan, W. Y. Tam, D. L. Phillips, K.-W. Cheah, Adv. Funct. Mater. 2008, 18, 566. 


\section{WILEY-VCH}

[14] Y. Lei, Q. Liao, H. Fu, J. Yao, J. Am. Chem. Soc. 2010, 132, 1742.

[15] M. Roushan, X. Zhang, J. Li, Angew. Chem. 2012, 124, 451.

[16] M. Shimizu, K. Mochida, M. Katoh, T. Hiyama, J. Phys. Chem. C 2010, 114, 10004.

[17] A. Camposeo, F. D. Benedetto, R. Cingolani, D. Pisignano, Appl. Phys. Lett. 2009, 94, 043109.

[18] D. P. Puzzo, M. G. Helander, P. G. O’Brien, Z. Wang, N. Soheilnia, N. Kherani, Z. Lu, G. A. Ozin, Nano Lett. 2011, 11, 1457.

[19] F. J. Aparicio, G. Lozano, I. Blaszczyk-Lezak, Á. Barranco, H. Míguez, Chem. Mater. 2010, 22,379 .

[20] R. J. Barbour, P. A. Dalgarno, A. Curran, K. M. Nowak, H. J. Baker, D. R. Hall, N. G. Stoltz, P. M. Petroff, R. J. Warburton, J. Appl. Phys. 2011, 110, 053107.

[21] H. Zhang, Y. Dai, H. You, D. Ma, Opt. Quantum Electron. 2008, 39, 1319.

[22] O. Deparis, C. Vandenbem, J. P. Vigneron, Opt. Lett. 2007, 32, 686.

[23] J. R. Sánchez-Valencia, A. Borrás, A. Barranco, V. J. Rico, J. P. Espinós, A. R. González-Elipe, Langmuir 2008, 24, 9460.

[24] J. R. Sánchez-Valencia, I. Blaszczyk-Lezak, J. P. Espinós, S. Hamad, A. R. GonzálezElipe, A. Barranco, Langmuir 2009, 25, 9140.

[25] J. R. Sánchez-Valencia, J. Toudert, L. González-García, A. R. González-Elipe, A. Barranco, Chem. Commun. 2010, 46, 4372.

[26] P. Castillero, J. R. Sánchez-Valencia, M. Cano, J. M. Pedrosa, J. Roales, A. Barranco, A. R. González-Elipe, ACS Appl. Mater. Interfaces 2010, 2, 712.

[27] Y. Oulad-Zian, J. R. Sanchez-Valencia, J. Parra-Barranco, S. Hamad, J. P. Espinos, A. Barranco, J. Ferrer, M. Coll, A. Borras, Langmuir 2015, 31, 8294.

[28] T. Kasputis, A. Pieper, K. B. Rodenhausen, D. Schmidt, D. Sekora, C. Rice, E. Schubert, M. Schubert, A. K. Pannier, Acta Biomater. 2015, 18, 88. 


\section{WILEY-VCH}

[29] K. B. Rodenhausen, R. S. Davis, D. Sekora, D. Liang, A. Mock, R. Neupane, D.

Schmidt, T. Hofmann, E. Schubert, M. Schubert, J. Colloid Interface Sci. 2015, 455, 226.

[30] K. B. Rodenhausen, D. Schmidt, T. Kasputis, A. K. Pannier, E. Schubert, M. Schubert, Opt. Express 2012, 20, 5419.

[31] M. Oliva-Ramirez, A. Barranco, M. Löffler, F. Yubero, A. R. González-Elipe, ACS Nano 2016, 10, 1256.

[32] D. P. Singh, S. Kumar, J. P. Singh, $R S C A d v$ 2015, 5, 31341.

[33] J. R. Sanchez-Valencia, J. Toudert, A. Borras, A. Barranco, R. Lahoz, G. F. de la Fuente, F. Frutos, A. R. Gonzalez-Elipe, Adv. Mater. 2011, 23, 848.

[34] J. R. Sánchez-Valencia, J. Toudert, A. Borras, C. López-Santos, A. Barranco, I. O. Feliu, A. R. González-Elipe, Plasmonics 2010, 5, 241.

[35] S. H. Lee, H. Jin, D.-Y. Kim, K. Song, S. H. Oh, S. Kim, E. F. Schubert, J. K. Kim, Opt. Express 2014, 22, A867.

[36] A. Barranco, A. Borras, A. R. Gonzalez-Elipe, A. Palmero, Prog. Mater. Sci. 2016, $76,59$.

[37] Y. He, Y. Zhao, Nanoscale 2011, 3, 2361.

[38] D. Gall, 2011, p. 81040V-81040V-7.

[39] J. L. Plawsky, J. K. Kim, E. F. Schubert, Mater. Today 2009, 12, 36.

[40] C. M. Zhou, H. F. Li, D. Gall, Thin Solid Films 2008, 517, 1214.

[41] J. J. Steele, M. J. Brett, J. Mater. Sci. Mater. Electron. 2007, 18, 367.

[42] R. Vogel, P. Meredith, M. D. Harvey, H. Rubinsztein-Dunlop, Spectrochim. Acta. A. Mol. Biomol. Spectrosc. 2004, 60, 245.

[43] J. Loerke, F. Marlow, Adv. Mater. 2002, 14, 1745.

[44] S. A. Tomás, S. Stolik, R. Palomino, R. Lozada, C. Persson, I. Pepe, A. F. da Silva, J. Appl. Phys. 2005, 98, 073516. 


\section{WILEY-VCH}

[45] R. Vogel, P. Meredith, I. Kartini, M. Harvey, J. D. Riches, A. Bishop, N. Heckenberg, M. Trau, H. Rubinsztein-Dunlop, ChemPhysChem 2003, 4, 595.

[46] M. Oliva-Ramirez, L. González-García, J. Parra-Barranco, F. Yubero, A. Barranco, A. R. González-Elipe, ACS Appl. Mater. Interfaces 2013, 5, 6743.

[47] S. Colodrero, M. Ocaña, H. Míguez, Langmuir 2008, 24, 4430.

[48] Y. S. Zhao, H. B. Fu, F. Q. Hu, A. D. Peng, W. S. Yang, J. N. Yao, Adv. Mater. 2008, $20,79$.

[49] J. R. Sánchez-Valencia, F. J. Aparicio, J. P. Espinós, A. R. Gonzalez-Elipe, A. Barranco, Phys. Chem. Chem. Phys. 2011, 13, 7071.

[50] J. R. Lakowicz, Ed., Principles of Fluorescence Spectroscopy, Springer US, Boston, MA, 2006.

[51] F. J. Aparicio, M. Holgado, A. Borras, I. Blaszczyk-Lezak, A. Griol, C. A. Barrios, R. Casquel, F. J. Sanza, H. Sohlström, M. Antelius, A. R. González-Elipe, A. Barranco, Adv. Mater. 2011, 23, 761.

[52] N. Tessler, G. J. Denton, R. H. Friend, Nature 1996, 382, 695.

[53] S.-H. Cao, W.-P. Cai, Q. Liu, Y.-Q. Li, Annu. Rev. Anal. Chem. 2012, 5, 317.

[54] H. Aouani, O. Mahboub, E. Devaux, H. Rigneault, T. W. Ebbesen, J. Wenger, Nano Lett. 2011, 11, 2400.

[55] M. Macias-Montero, A. N. Filippin, Z. Saghi, F. J. Aparicio, A. Barranco, J. P. Espinos, F. Frutos, A. R. Gonzalez-Elipe, A. Borras, Adv. Funct. Mater. 2013, 23, 5981.

[56] S. Chan, P. M. Fauchet, Appl. Phys. Lett. 1999, 75, 274. 